\title{
Bitter Taste Receptor as a Therapeutic Target in Orthopaedic Disorders
}

This article was published in the following Dove Press journal:

Drug Design, Development and Therapy

\author{
Weyland Cheng $\mathbb{B D}^{1,2}$ \\ Manye Yao' \\ Fangna Liu' \\ 'Department of Orthopaedic Surgery, \\ Children's Hospital Affiliated to \\ Zhengzhou University, Henan Children's \\ Hospital, Zhengzhou Children's Hospital, \\ Zhengzhou, Henan, People's Republic of \\ China; ${ }^{2}$ Henan Provincial Key Laboratory \\ of Children's Genetics and Metabolic \\ Diseases, Children's Hospital Affiliated to \\ Zhengzhou University, Henan Children's \\ Hospital, Zhengzhou Children's Hospital, \\ Zhengzhou, Henan, People's Republic of \\ China
}

\begin{abstract}
Non-gustatory, extraoral bitter taste receptors (T2Rs) are G-protein coupled receptors that are expressed throughout the body and have various functional responses when stimulated by bitter agonists. Presently, T2Rs have been found to be expressed in osteoclasts and osteocytes where osteoclasts were capable of detecting bacterial quorumsensing molecules through the T2R38 isoform. In the innate immune system, stimulating T2Rs induces anti-inflammatory and anti-pathogenic effects through the phospholipase C/ inositol triphosphate pathway, which leads to intracellular calcium release from the endoplasmic reticulum. The immune cells with functional responses to T2R activation also play a role in bone inflammation and orthopaedic disorders. Furthermore, increasing intracellular calcium levels in bone cells through T2R activation can potentially influence bone formation and resorption. With recent studies finding $\mathrm{T} 2 \mathrm{R}$ expression in bone cells, we examine the potential of targeting this receptor to treat bone inflammation and to promote bone anabolism.
\end{abstract}

Keywords: TAS2R, calcium signaling, osteoblasts, drug target, bone inflammation

\section{Introduction}

G protein-coupled receptors (GPCRs) are a large, diverse group of protein receptors that can vary widely in structure and function. Based on the GRAFS classification system, the GPCR superfamily can be categorized into five main families: Glutamate, Rhodopsin, Adhesion, Frizzled/Taste2, and Secretin. ${ }^{1}$ Bitter taste receptors (T2Rs) belong to the Frizzled/Taste 2 family and consist of 25 functional isoforms in humans. T2Rs were originally thought to be primarily functional in the gustatory system where they served the purpose of bitter taste detection and acted as a warning mechanism against the ingestion of noxious substances.

T2Rs have an attached heterotrimeric G-protein subunit consisting of $\mathrm{G} \alpha$ gustducin, G 33 and Gy13. Activation of the receptor causes the separation of the G-protein subunits into Ga-gustducin and the $\beta \gamma$-gustducin dimer, which subsequently leads to second messengers. ${ }^{2,3} \beta \gamma$-gustducin induces the phospholipase $C \beta 2$ (PLC 32 )/inositol-1,4,5-triphosphate (IP3) signaling pathway resulting in the activation of type III IP3 receptors (IP3R) on the endoplasmic reticulum (ER) and the release of calcium stored in the ER. ${ }^{4}$ On the other hand, $\alpha$-gustducin stimulates phosphodiesterase (PDE), which hydrolyzes cAMP thereby decreasing intracellular cAMP levels. ${ }^{5,6}$

In the past 15 years, extraoral (non-gustatory) T2Rs were discovered to be expressed and functional in multiple regions of the body including the respiratory,
Correspondence: Weyland Cheng

Tel $+86 \quad 8502758200$

Email wey_c@hotmail.com
Drug Design, Development and Therapy 2021:15 895-903

895

ubmit your manuscript

DovePress

in $\square$ 
gastrointestinal, endocrine, genitourinary, immune, cardiovascular and neurological system. ${ }^{7,8}$ When stimulated, T2Rs can have anti-pathogenic effects in the lungs, antiinflammatory effects in immune cells, and regulate glucose homeostasis in the gastrointestinal tract. ${ }^{9-13}$ A majority of extraoral $\mathrm{T} 2 \mathrm{R}$ research has been invested in the treatment of respiratory infections and inflammation where bitter agonists are capable of inducing antipathogenic nitric oxide production, promoting antimicrobial peptide secretion (AMP) and increasing ciliary beat frequency in respiratory cells. ${ }^{14}$

Minimal studies have investigated the role of T2R activation in orthopaedic disorders and bone. Presently, two in vitro studies have discovered the expression of T2Rs in bone cells. Gaida et al (2016) obtained tissue samples from patients with osteomyelitis and observed the expression of T2R38 on phagocytic cells such as neutrophils, macrophages, and multinucleated osteoclastic giant cells. ${ }^{15}$ The group found that these cells were able to detect bacterial biofilm through the T2R38-agonising bacterial quorum-sensing molecule, acyl-homoserine lactone (AHL). Lund et al (2013) investigated the presence of T2R46 on osteocytes differentiated from mesenchymal stromal cells. ${ }^{16}$ The study focused on the function of human mesenchymal stromal cells and human aortic smooth muscle cells whereas their measurements on TAS2R46 expression in osteocytes were conducted to confirm that expression was not lost after mesenchymal stromal cell differentiation. Wang et al (2019) also speculated the promotion of bone formation and osteoblast proliferation through the activation of $\mathrm{T} 2 \mathrm{R} 7$ by $\mathrm{Al}^{3+}$ based on their study of metal ions activating the T2R7 isoform. ${ }^{17}$ Consequently, we examine the implications of T2Rs in bone and its potential as a therapeutic target.

\section{Relevant Studies on Bitter Taste Receptors \\ Inflammation and Infection}

The ability for T2Rs to detect bacterial products was first shown using the gram-negative bacteria, Pseudomonas aeruginosa, where T2R38-dependent intracellular calcium $\left(\left[\mathrm{Ca}^{2+}\right]_{\mathrm{i}}\right)$ and nitric (NO) levels in sinonasal epithelial cells were increased in response to the bacterial secretion of AHL. ${ }^{11}$ Although chronically high levels of NO production through inducible nitric oxide synthase (iNOS) induced by cytokines or microbial products can be proinflammatory, acute or low levels of NO can have anti- inflammatory and antipathogenic effects. ${ }^{18}$ Similar studies followed, exhibiting the wide binding range of T2Rs to both gram-positive and gram-negative bacteria products across different cells types. Quinolones from $P$. aeruginosa were shown to activate T2Rs in T2Rtransfected HEK293T cells as well as increase calcium and NO through T2R pathways in primary airway cells. $^{19} \mathrm{~N}$-(3-oxododecanoyl)-L-homoserine lactone (AHL-12) from $P$. aeruginosa was found to bind with T2R38 expressed in myeloid cells. ${ }^{20}$ Gram-positive bacteria including Bacillus cereus, Staphylococcus aureus, and Staphylococcus epidermidis elicited NO production through the T2R pathway in the upper epithelial airway. $^{21-23}$ It has been further shown that human monocyte-derived unprimed macrophages exhibited low-level NO production through endothelial NOS (eNOS) and neuronal NOS (nNOS) isoforms. ${ }^{24}$

Tran et al (2018) discovered expression of T2R38 in human peripheral mononuclear blood cells (PMBCs) consisting of lymphocytes, monocytes, and granulocytes. ${ }^{25}$ The authors found higher levels of T2R expression in memory and effector T-cells compared to naïve T-cells. Furthermore, stimulation of T-cells with bitter agonists resulted in increased T2R38 expression. The application of the T2R38 agonist goitrin to PMBCs resulted in an increase in calcium concentration and the inhibition of tumour necrosis factor alpha (TNF- $\alpha$ ) secretion.

Stimulating T2Rs in cells can also mediate histamine and prostaglandin production. In LPS-induced blood leukocytes, T2R activation using chloroquine inhibited the release of prostaglandin E2 $\left(\mathrm{PGE}_{2}\right)$ and several proinflammatory factors including interleukin (IL)-1 $\beta$, IL-2, IL-4, IL-5, IL-10, IL-17, granulocyte stimulating factor (G-CSF) and granulocyte-macrophage colony-stimulating factor (GM-CSF). ${ }^{12}$ Both bitter agonists denatonium and chloroquine inhibited TNF- $\alpha$, IL-13 and monocyte chemoattractant protein-1 (MCP-1). In another study, activation of T2Rs inhibited histamine and prostaglandin D2 $\left(\mathrm{PGD}_{2}\right)$ in both human cord blood-derived mast cells and the HMC1.2 cell line via the inhibition of IgE-dependent mast cell activation. ${ }^{26}$

\section{Genetic Polymorphisms}

Genetic and phenotypic variants among the 25 T2R isoforms have been observed, most notably that of T2R38. Genetic variability of the T2R gene (TAS2R) can influence taste perception where the human population can be categorized into non-tasters, who are unable to detect 
certain bitter flavors; intermediate tasters; and supertasters, who are able to detect exceedingly small quantities of bitter compounds. ${ }^{27-31}$ In studying the genotypephenotype relationship of bitter taste perception, Kim et al (2003) identified two predominant haplotypes based on three single-nucleotide polymorphisms (SNPs) in a major locus on chromosome 7q (rs714598, rs1726866, rs 10246939), which were relevant to propylthiocarbamide (PTC) taste sensitivity. ${ }^{30}$ The SNPs were positioned at the encoding amino acids 49, 262, and 296 of the TAS2R38 allele and named according to its variants: proline, alanine, and valine (PAV) for the taster phenotype and alanine, valine, and isoleucine (AVI) for the non-taster phenotype. The authors found that PAV homozygotes (PAV/PAV) had the highest sensitivity to PTC whereas PAV heterozygotes (PAV/AVI) had a significantly lower sensitivity and AVI homozygotes (AVI/AVI) had the lowest sensitivity.

Genetic variants are not just limited to affecting taste perception, but can play a role in respiratory immune responses. A study by Lee et al (2012) found that the cellular response of human sinonasal epithelial cells (derived from PAV/PAV supertasters) to T2R38 agonists had the highest elevation of calcium in comparison to cells derived from AVI/PAV and AVI/AVI individuals, both of which displayed minimal calcium elevation. ${ }^{11}$ Furthermore, T2R38-stimulated PAV/PAV cells showed higher NO production and ciliary beat frequency, resulting in significantly enhanced bactericidal activity in vitro compared to cells from the other two polymorphisms. In light of the dependence of T2R38 function on the PAV haplotype, studies have also been performed to investigate the effects of TAS2R38 polymorphisms on chronic rhinosinusitis (CRS). A collection of these findings indicated that TAS2R38 PAV/PAV supertasters were less likely to require surgical intervention for CRS. ${ }^{32-35}$ Following, a study on bacterial cultures derived from nonpolypoid CRS patient swabs revealed that in vitro biofilm formation was inversely correlated with PTC taste sensitivity. ${ }^{36}$ Likewise, Rom et al (2017) found that the non-taster TAS2R38 genotype was a predictor for the presence of culturable bacteria in the sinus cavity. ${ }^{37}$

\section{Implications in Bone Calcium Signaling}

Intracellular calcium in bone cells can be modulated through the release of $\mathrm{Ca}^{2+}$ from the ER or through extracellular $\mathrm{Ca}^{2+}$ influx through voltage gated calcium channels. ${ }^{38}$ T2R activation influences intracellular calcium through the PLC/IP3 pathway and calcium signaling vitally influences multiple pathways in bone cells. The PLC/IP3 pathway is a known mechanism for $\mathrm{Ca}^{2+}$ mobilization in bone homeostasis as seen with the activation of the calcium-sensing receptor (CaSR), a GPCR found in both osteoblasts and osteoclasts. CaSR in response to extracellular $\mathrm{Ca}^{2+}$ is thought to regulate parathyroid secretion and parathyroid hyperplasia. Interestingly, some studies have failed to detect the presence of CaSR in certain bone cells and have surmised that a similar a cationsensing mechanism is present. ${ }^{39}$ This notion can be observed in studies on strontium ranelate, which can mediate cell differentiation, enhance matrix mineralization, reduce receptor activator of nuclear factor $\kappa B$ (RANK) ligand (RANKL) expression and increase osteoprotegerin expression in osteoblasts through CaSR activation. ${ }^{40}$ Despite these findings on CaSR activation, $\mathrm{Sr}^{2+}$ has still been observed to enhance proliferation in osteoblasts isolated from $\mathrm{CaSR}-/$ - mice, indicating another mechanism at play. ${ }^{41}$ Likewise, osteoblasts isolated from CaSR knockout mice were also responsive to extracellular calcium. ${ }^{41,42}$

Proposed cation-sensing alternatives to CaSR consist of ryanodine receptors; calcyclin, an intracellular calciumbinding protein; GPRC6A, a widely expressed GPCR; and CaSR splice variants. ${ }^{39,43}$ An additional potential receptor that can be examined is the T2R7 isoform, where Wang et al (2019) found that several metal ions including calcium, zinc, magnesium, copper, manganese and aluminum, elicited a dose-dependent response in TAS2R7 transfected human embryonic kidney cells. ${ }^{17}$ In accordance, calcium salts are perceived to have taste properties that are largely associated with bitterness. ${ }^{44}$

In osteoblasts, increasing $\left[\mathrm{Ca}^{2+}\right]_{\mathrm{i}}$ can induce cell proliferation through the calmodulin kinase (CaMK)dependent pathway. In this pathway, calcium activates calmodulin (CaM) leading to the activation of $\mathrm{CaM}$ kinase II (CaMKII) and calcineurin (Cn). ${ }^{38} \mathrm{CaM}$ has multiple functions in regulating the activity of calcium pumps and channels in combination with $\mathrm{Ca}^{2+}$ and has a critical role in osteoblast differentiation. $\mathrm{Ca}^{2+} / \mathrm{CaM}$ activates $\mathrm{Cn}$ which binds to and dephosphorylates nuclear factor of activated T cells (NFAT) transcription factors. NFAT is then able to translocate into the nucleus where it regulates gene expression (Figure 1). ${ }^{38}$ Stimulation of CaSRs through extracellular calcium can also activate multiple targets including phospholipase 


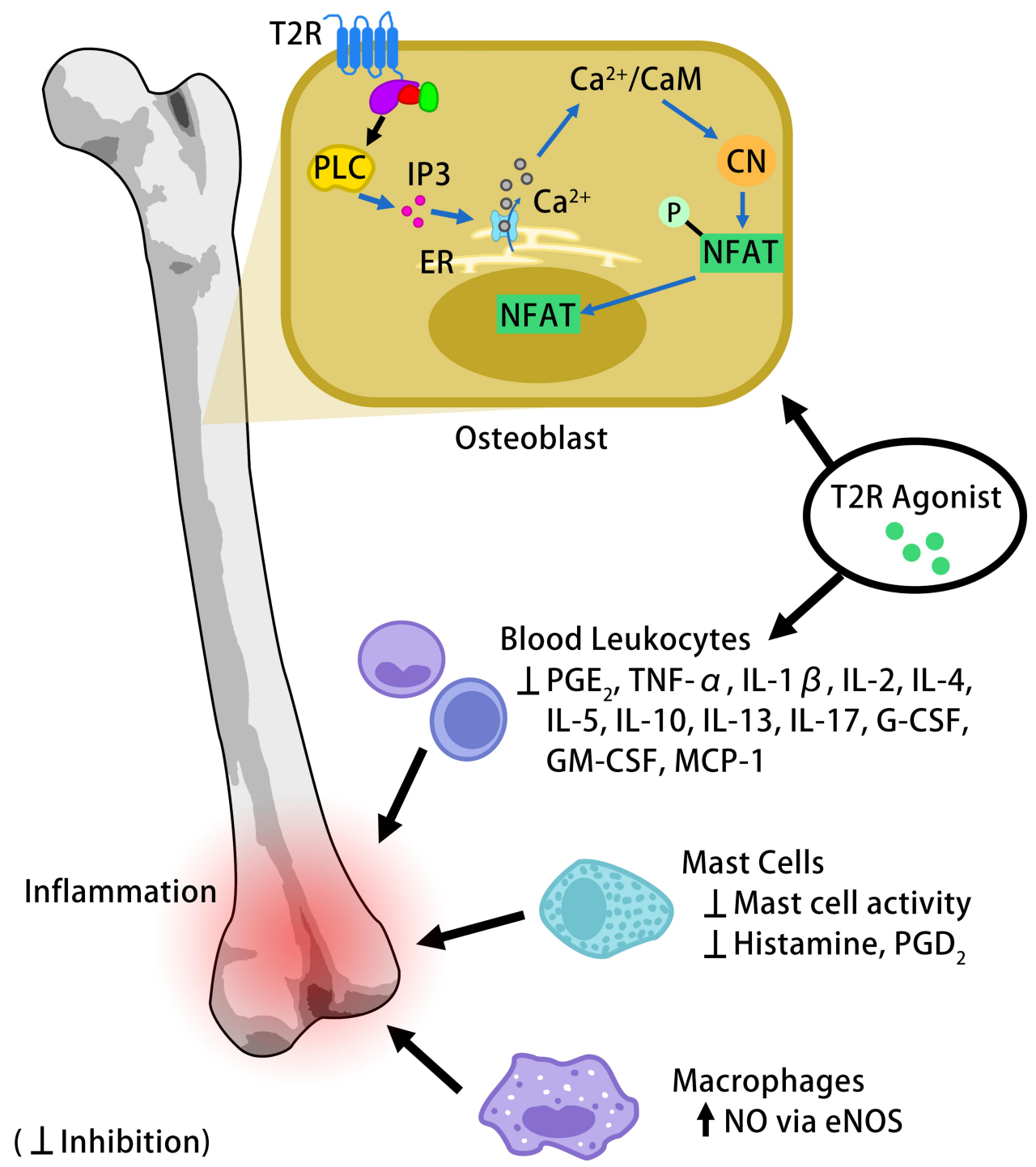

Figure I Potential functional effects of T2R activation in bone. Osteoblast formation and osteoclastogenesis can be modulated through the PLC/IP3 signaling pathway, which leads to the release of calcium stored in the ER. T2R activation inhibits pro-inflammatory activity in the immune cells that migrate to inflammatory sites.

$\mathrm{C}$ and protein kinase $\mathrm{C}$, extracellular signal-regulated kinases, c-Jun N-terminal kinase, AKT and cAMP/protein kinase A pathways with the functional effects of regulating bone cell proliferation, differentiation and apoptosis. $^{43}$

Calcium levels also influence bone resorption where cytosolic $\mathrm{Ca}^{2+}$ oscillations occur during RANKL-mediated osteoclastogenesis. Activation of the PLC/IP3 pathway through extracellular stimuli is a common mechanism for $\mathrm{Ca}^{2+}$ mobilization in osteoclasts. Binding of RANKL to RANK eventually leads to the activation of PLCY by spleen tyrosine kinase, the production of IP3 and the release of $\mathrm{Ca}^{+}$from the ER. ${ }^{45}$ In receptor agonist concentrations of physiological conditions, calcium influx through store-operated $\mathrm{Ca}^{2+}$ entry (SOCE) likely plays a role in maintaining $\mathrm{Ca}^{2+}$ oscillation. High levels of extracellular $\mathrm{Ca}^{2+}$ can also increase $\left[\mathrm{Ca}^{2+}\right]_{\mathrm{i}}$ levels through PLC activation and regulate bone resorption activity. ${ }^{45}$

Future studies in bone cells can thus examine the effects of T2R activation in regulating downstream pathways and bone metabolism. Many of these effects can be already be observed in copious studies using both natural and synthetic bitter bioactives on bone cells. For example, a study by Yamaguchi et al (2007) cultured mouse bone marrow cells in the presence of parathyroid hormone (PTH) to induce osteoclastogenesis. ${ }^{46}$ The authors found that the flavonoids quercetin, myricetin, kaempferol, isorhamnetin and curcumin inhibited osteoclast-like cell formation whereas hesperidin and astaxanthin did not. Culture with PTH also caused a decrease in diaphyseal 
calcium content, which was prevented by quercetin, myricetin, kaempferol and isorhamnetin. Correspondingly, hesperidin or astaxanthin are known to be neutral tasting flavonoids whereas quercetin, myricetin, kaempferol, isorhamnetin and curcumin are bitter, although curcumin has presently not been shown to activate any T2R isoforms. ${ }^{47}$

\section{Osteoimmunology}

Inflammatory conditions can cause imbalances in bone homeostasis, which is observed in patients with osteoporosis, osteoarthritis, implant failure/aseptic implant loosening, bone tumors and osteoarticular infections. Osteoclasts and pro-inflammatory cytokines are two major factors that link inflammatory diseases to bone resorption. Inflammatory diseases, such as rheumatoid arthritis, systemic lupus erythematosus, inflammatory bowel disease and chronic obstructive pulmonary disease, have all been associated with osteoporosis where RANKL, M-CSF and pro-inflammatory cytokines are major contributors to bone resorption. ${ }^{48}$ Aseptic loosening of total joint replacements is caused by chronic inflammation and osteolysis, which develops in response to particulate debris at the implantbone interface. This inflammatory response is manifested by macrophages, giant cells, neutrophils, lymphocytes and osteoclasts in addition to the secretion of pro-inflammatory factors such as TNF, RANKL, IL-6, IL-1, and IL- $11 .{ }^{49}$ Inflammation in skeletal tumor metastases also plays a large role in osteolysis and bone resorption. The tumor microenvironment consists of several leukocyte populations, including neutrophils, eosinophils, basophils, monocytes/macrophages, dendritic cells, natural killer (NK) cells and lymphocytes, that contribute to persistent inflammation and tumor progression. ${ }^{50,51}$ The bone marrow is rich in myeloid precursors and differentiated immune cells where the signaling mechanisms of these cells can promote proinflammatory cell activity in the bone microenvironment. ${ }^{51}$ Skeletal metastasis thus affects bone homeostasis through cancer colonization and the perpetuation of inflammation.

As T2R activation has been demonstrated to reduce inflammatory factors in various immune cells, bitter agonists can thus be considered for prophylactic or therapeutic use. In the event of a bone injury, immune cells such as macrophages, NK cells, B-lymphocytes and T-lymphocytes play an essential role in the migration of immune cells and multipotential stromal cells (MSCs), osteoclastogenesis, clearance of debris at the injured site, and the licensing of MSCs. ${ }^{52}$ Pro-inflammatory cytokines and chemokines including TNF- $\alpha$, IL-1, IL-6, IL-8, MCP1, CCL5, CCL3, chemokine (C-X-C motif) ligand 7 (CXCL7), stromal derived factor-1 (SDF-1) and RANKL all contribute to this inflammatory process.

The NOS isoform expressed in bone is predominantly eNOS where it plays a regulatory role in osteoblast activity and bone anabolism. ${ }^{53}$ During inflammation, proinflammatory cytokines, such as IL-1 and TNF- $\alpha$ can trigger NO production through the transcription of iNOS. The iNOS pathway promotes IL-1 induced inflammatory bone resorption. High concentrations of NO induced by activation of the iNOS pathway through interferon $\gamma$ (IFN$\gamma$ ) combined with IL-1 and/or TNF- $\alpha$, however, can inhibit osteoclast formation and activity. High NO concentration can also inhibit osteoblast growth and differentiation.

Production of prostaglandins, such as PGE2, in osteoblasts and osteoclasts is largely dependent on the cyclooxygenase-2 (COX-2) induction. Understanding the role of prostaglandins in human skeletal metabolism is challenging due to cells having multiple G-protein linked receptors and exhibiting opposing functional effects. ${ }^{54}$ Prostaglandin induction through differing pathways can result in differences in the way osteoclasts or osteoblasts differentiate. PGE2 is believed to favor bone formation when administered intermittently and bone loss when administered continuously. ${ }^{55}$

Mast cells also secrete several mediators that can exert osteocatabolic or osteoprotective effects in bone and may contribute to orthopaedic disorders such as rheumatoid arthritis, osteoarthritis and osteoporosis. ${ }^{56}$ Patients with these disorders tend to have higher mast cells numbers. Mast cell mediators such as histamine, TNF- $\alpha$, IL-6, PGE2 and CCL3 promote osteoclastogenesis whereas IL-1, IL-10, TNF- $\alpha$ and MCP- 1 can inhibit osteoblast activity. Other mast cell mediators are osteoprotective where transforming growth factor- $\beta$ (TGF- $\beta$ ) stimulates osteoblast activity and IL-12 and IFN- $\gamma$ can reduce osteoclastogenesis. ${ }^{56}$

Previously, we showed that T2R agonists are capable of regulating many of the aforementioned proinflammatory factors present in bone inflammation. Thus, targeting this receptor in immune cells could be a method of inhibiting the production of pro-inflammatory cytokines and chemokines, prostaglandins, and histamine.

\section{Osteoarticular Infections}

The formation of biofilms in osteomyelitis and septic arthritis patients can make the infection difficult to treat. Complications in osteomyelitis can lead to osteonecrosis, 
gangrene, septic arthritis, and impaired growth in children. Therefore, early and accurate prognosis, reducing the need for surgical treatment, preventing or treating biofilm formation, and limiting osteonecrosis are essential factors for preferable outcomes. Dead bone or orthopedic implantations can act as a nonliving surface for the attachment of bacteria, resulting in the formation of biofilms. ${ }^{57}$ Bacterial biofilms have also been shown to be the reason for failed antibiotic treatments in adult patients with orthopedic implants. ${ }^{58,59}$

The ability for AHL to trigger T2R38 signifies the conceivability for bone cells to detect and functionally react to bacterial biofilm. ${ }^{15}$ Comparatively, with regards to respiratory cells, Lee et al (2014) stimulated T2Rs in human solitary chemosensory cells and discovered that neighboring epithelial cells experienced increases in $\left[\mathrm{Ca}^{2+}\right.$ ]$_{\mathrm{i}}$ by receiving calcium ions through the gap junction. The increase in calcium levels triggered the secretion of two AMPs, human $\beta$-defensins 1 and $2 .{ }^{60}$ Similarly, osteoblasts have been shown to produce human $\beta$-defensin-3 (HBD3) and human $\beta$-defensin-2 (HBD2) in the presence of bacteria. ${ }^{61,62}$ The induction of HBD3 has been found to be dependent on toll-like receptors- 2 and $-4 .{ }^{62}$ The mechanism for HBD production could be in part through the activation of $\mathrm{p} 38$ mitogen-activated protein kinase (MAPK) and nuclear factor- $\mathrm{KB}(\mathrm{NF}-\kappa \mathrm{B})$ pathways. ${ }^{63}$ The mechanism of action for HBD2 secretion in osteoblasts has yet to be determined, but the presence of toll-like receptors on osteoblasts does not preclude the involvement of other receptors, such as the nucleotide-binding oligomerization domain and T2Rs. ${ }^{61}$

Genetic polymorphisms may also affect outcomes in osteoarticular infections. Factors such as the severity of infection, number of surgeries required, recurrence or the formation of bacterial biofilm can be influenced by innate responses related to $\mathrm{T} 2 \mathrm{R}$ variants, as seen in CRS patients with T2R38 polymorphisms. ${ }^{32-37}$ Thus, along with the anti-inflammatory effects of T2R agonists, potential topics of study for osteoarticular infections include the release of AMPs in osteoblasts through T2R stimulation and the consequence of genetic variants.

\section{Potential for Drug Development}

Research in targeting T2Rs for clinical use is still in its infancy where in vivo studies on the efficacy of T2R agonists have mostly been conducted in animal studies. Challenges for the development of T2R-agonising drugs include the poor bioavailability of natural bitter bioactives as seen in bitter flavonoids. ${ }^{64}$ In addition, re-purposed synthetic drugs may lack sufficient potency and the pharmaceutical effects of these drugs may be attributed to other mechanisms. T2R isoforms can also range from being broadly tuned to narrowly tuned and it is suggested that many bitter compounds only function as a partial agonist. ${ }^{47} \mathrm{~A}$ bitter compound can also be agonistic to one T2R isoform, but antagonistic to another. Despite these limitations, there has been some progress in the screening and development of more potent agonists as well as progress in the clinical application of T2Rtargeting drugs.

Research on T2R agonists can lead to the discovery of novel drug candidates. Recently, Kim et al (2020) screened for T2R5 agonists using human airway smooth muscle to identify potent compounds. The authors identified a highly potent compound $\left(\mathrm{EC}_{50} \sim 120 \mathrm{nM}\right)$ in 1,10-Phenanthroline -5,6-dione, which had a >1000-fold improvement in potency over other compounds. 110-Phenanthroline -5,6-dione also had maximal efficacy based on $\left[\mathrm{Ca}^{2+}\right]_{\mathrm{i}}$ release in airway smooth muscle cells.

Regarding the clinical usage of T2R agonists, MPAzeFlu (Dymista ${ }^{\circledR}$; spray of azelastine/fluticasone propionate) is a relatively new pharmaceutical drug used to treat allergic rhinitis. It has been found to be more effective than azelastine hydrochloride (AZE) or fluticasone propionate alone in reducing cytokine secretion and reducing eosinophil survival. ${ }^{65}$ Upon investigation towards the mechanism of MP-AzeFlu, Ekstedt et al (2020) discovered that the dilatory effects of MP-AzeFlu were independent of the common airway dilatory pathways involving histamine receptors (H1-, H2- and H3-), prostaglandins, cAMP and cGMP. ${ }^{66}$ When examining pre-contracted murine airways, the authors found that MP-AzeFlu and AZE had dilatory effects similar to that of T2R agonists, suggesting that MP-AzeFlu functioned through bitter taste receptor activation.

Deloose et al (2017) studied the effects of the bitter tastant denatonium benzoate on antral motility and hunger ratings in healthy volunteers. ${ }^{67}$ Upon intragastric administration of denatonium benzoate, women were found more likely to have decreased motilin plasma concentrations and a decrease in hunger ratings with increased satiety. The same group performed a similar study to evaluate the gastrointestinal effects of the bitter tastant quininehydrochloride $(\mathrm{QHCl})$ in healthy female volunteers. ${ }^{68}$ Results showed that the intragastric administration of $\mathrm{QHCl}$ suppressed the increase of motilin and ghrelin 
plasma levels as well as reduced the fluctuation of antral motility. Thus, these clinical studies demonstrated the potential of T2Rs as targets for interdigestive motility.

Researchers have found that T2Rs can also be expressed in the skin where bitter agonists induced calcium influx in keratinocytes and initiated differentiation through the expression of keratin 10, involucrin and transglutaminase. $^{69}$ The same group noted in a patent that the topical application of a cream with T2R ligands relieved itchiness and redness in a case of atopic eczema. ${ }^{70}$

Consequently, three different routes of T2R agonist delivery have been presented in the forms of inhalation, intragastric infusion and topical administration. Drug delivery methods for bone can also be diverse as oral administration of T2R-targeting drugs may result in poor bioavailability or induce adverse effects at high dosages. Viable methods of drug administration to target bone include nasal delivery, parenteral routes and local implantation. ${ }^{71}$ In orthopaedic conditions that require surgical debridement procedures, such as osteomyelitis, antibiotic-loaded implants or biodegradable drug delivery scaffolds can be used to fill tissue voids and facilitate the healing process. $^{72}$ In this manner, local implanted delivery of $\mathrm{T} 2 \mathrm{R}$ agonists could potentially improve anti-microbial and anti-inflammatory effects in order to fight off infection and prevent bone resorption. More advanced methods such as an osteotropic drug delivery system using nanoparticles to passively target bone are also potential research topics in delivering T2R ligands. ${ }^{71,73}$

\section{Conclusion}

In this article, we provided an insight to the potential role of T2Rs in orthopaedics and bone cells. Through the T2R pathway, it is currently known that bitter agonists can reduce or inhibit inflammatory effects and regulate calcium signaling through second messengers via the separation of G-protein subunits. Anti-inflammatory medications are widely used for various orthopaedic conditions since inflammation plays a fundamental role in bone resorption. T2R agonists can be studied as an alternative to nonsteroidal anti-inflammatory medications in order to avoid side effects or as additive treatment to improve efficacy. Future directions in understanding the role of T2Rs in bone homeostasis consist of examining the functional responses of T2R activation in bone cells.

Specific research considerations also emerge, such as the ability for osteoblasts to detect AHL in bacterial biofilm through the T2R pathway and the ability for these osteoblasts to release HBD's in response.
Furthermore, genetic TAS2R polymorphisms in patients with osteoarticular infections could play a factor in bacterial resistance or in the frequency of surgical intervention. Knowledge of TAS2R polymorphisms could assist physicians in predicting the course of the disease and facilitate treatment decisions. Lastly, controversy over the presence of a cation-sensing mechanism in osteoblasts similar to that of CaSR could possibly be explained by functional T2Rs where metal ions are able to trigger the T2R7 isoform. Targeting a novel cationsensing mechanism in osteoblasts and osteoclasts may be a method to simultaneously promote bone formation and inhibit bone resorption in order to increase bone mass for orthopaedic disorders such as osteoporosis.

\section{Disclosure}

The authors report no conflicts of interest in this work.

\section{References}

1. Schioth HB, Fredriksson R. The GRAFS classification system of G-protein coupled receptors in comparative perspective. Gen Comp Endocrinol. 2005;142(1-2):94-101. doi:10.1016/j.ygcen.2004.12.018

2. Margolskee RF. Molecular mechanisms of bitter and sweet taste transduction. J Biol Chem. 2002;277(1):1-4. doi:10.1074/jbc.R100 054200

3. Chaudhari N, Roper SD. The cell biology of taste. J Cell Biol. 2010;190(3):285-296. doi:10.1083/jcb.201003144

4. Clapp TR, Stone LM, Margolskee RF, Kinnamon SC. Immunocytochemical evidence for co-expression of Type III IP3 receptor with signaling components of bitter taste transduction. BMC Neurosci. 2001;2:6. doi:10.1186/1471-2202-2-6

5. Lindemann B. Chemoreception: tasting the sweet and the bitter. Curr Biol. 1996;6(10):1234-1237. doi:10.1016/S0960-9822(96)00704-X

6. Kinnamon SC. Taste receptor signalling - from tongues to lungs. Acta Physiol (Oxf). 2012;204(2):158-168. doi:10.1111/j.1748-1716.2011. 02308.x

7. Jaggupilli A, Singh N, Upadhyaya J, et al. Analysis of the expression of human bitter taste receptors in extraoral tissues. Mol Cell Biochem. 2017;426(1-2):137-147. doi:10.1007/s11010-016-2902-z

8. Bloxham CJ, Foster SR, Thomas WG. A bitter taste in your heart. Front Physiol. 2020;11:431. doi:10.3389/fphys.2020.00431

9. Deshpande DA, Wang WC, McIlmoyle EL, et al. Bitter taste receptors on airway smooth muscle bronchodilate by localized calcium signaling and reverse obstruction. Nat Med. 2010;16(11):1299-1304. doi:10.1038/nm.2237

10. Dotson $\mathrm{CD}$, Zhang L, Xu H, et al. Bitter taste receptors influence glucose homeostasis. PLoS One. 2008;3(12):e3974. doi:10.1371/journal.pone. 0003974

11. Lee RJ, Xiong G, Kofonow JM, et al. T2R38 taste receptor polymorphisms underlie susceptibility to upper respiratory infection. J Clin Invest. 2012;122(11):4145-4159. doi:10.1172/JCI64240

12. Orsmark-Pietras C, James A, Konradsen JR, et al. Transcriptome analysis reveals upregulation of bitter taste receptors in severe asthmatics. Eur Respir J. 2013;42(1):65-78. doi:10.1183/090319 36.00077712

13. Shah AS, Ben-Shahar Y, Moninger TO, Kline JN, Welsh MJ. Motile cilia of human airway epithelia are chemosensory. Science. 2009;325 (5944):1131-1134. doi:10.1126/science.1173869 
14. Lee RJ, Cohen NA. Taste receptors in innate immunity. Cell Mol Life Sci. 2015;72(2):217-236.

15. Gaida MM, Dapunt U, Hansch GM, Bjarnsholt T. Sensing developing biofilms: the bitter receptor T2R38 on myeloid cells. Pathog Dis. 2016;74(3):ftw004. doi:10.1093/femspd/ftw004

16. Lund TC, Kobs AJ, Kramer A, et al. Bone marrow stromal and vascular smooth muscle cells have chemosensory capacity via bitter taste receptor expression. PLoS One. 2013;8(3):e58945. doi:10.1371/ journal.pone.0058945

17. Wang Y, Zajac AL, Lei W, et al. Metal ions activate the human taste receptor TAS2R7. Chem Senses. 2019;44(5):339-347. doi:10.1093/ chemse/bjz024

18. Zamora R, Vodovotz Y, Billiar TR. Inducible nitric oxide synthase and inflammatory diseases. Mol Med. 2000;6(5):347-373.

19. Freund JR, Mansfield CJ, Doghramji LJ, et al. Activation of airway epithelial bitter taste receptors by Pseudomonas aeruginosa quinolones modulates calcium, cyclic-AMP, and nitric oxide signaling. J Biol Chem. 2018;293(25):9824-9840. doi:10.1074/jbc.RA117.00 1005

20. Maurer S, Wabnitz GH, Kahle NA, et al. Tasting pseudomonas aeruginosa biofilms: human neutrophils express the bitter receptor T2R38 as sensor for the quorum sensing molecule N-(3-oxododecanoyl)-1-homoserine lactone. Front Immunol. 2015;6:369. doi:10.3389/fimmu.20 15.00369

21. Carey RM, Workman AD, Yan CH, et al. Sinonasal T2R-mediated nitric oxide production in response to Bacillus cereus. Am J Rhinol Allergy. 2017;31(4):211-215. doi:10.2500/ajra.2017.31.4453

22. Carey RM, Workman AD, Chen B, et al. Staphylococcus aureus triggers nitric oxide production in human upper airway epithelium. Int Forum Allergy Rhinol. 2015;5(9):808-813. doi:10.1002/alr.21568

23. Carey RM, Chen B, Adappa ND, et al. Human upper airway epithelium produces nitric oxide in response to Staphylococcus epidermidis. Int Forum Allergy Rhinol. 2016;6(12):1238-1244. doi:10.1002/alr.21837

24. Gopallawa I, Freund JR, Lee RJ. Bitter taste receptors stimulate phagocytosis in human macrophages through calcium, nitric oxide, and cyclic-GMP signaling. Cell Mol Life Sci. 2020;78(1):271-286. doi:10.1007/s00018-020-03494-y

25. Tran HTT, Herz C, Ruf P, Stetter R, Lamy E. Human T2R38 bitter taste receptor expression in resting and activated lymphocytes. Front Immunol. 2018;9:2949. doi:10.3389/fimmu.2018.02949

26. Ekoff M, Choi JH, James A, Dahlen B, Nilsson G, Dahlen SE. Bitter taste receptor (TAS2R) agonists inhibit IgE-dependent mast cell activation. J Allergy Clin Immunol. 2014;134(2):475-478. doi:10.10 16/j.jaci.2014.02.029

27. Drewnowski A, Henderson SA, Shore AB. Genetic sensitivity to 6-n-propylthiouracil (PROP) and hedonic responses to bitter and sweet tastes. Chem Senses. 1997;22(1):27-37. doi:10.1093/chemse/ 22.1.27

28. Bartoshuk LM, Duffy VB, Miller IJ. PTC/PROP tasting: anatomy, psychophysics, and sex effects. Physiol Behav. 1994;56(6):11 65-1171. doi:10.1016/0031-9384(94)90361-1

29. Whissell-Buechy D, Wills C. Male and female correlations for taster (P.T.C.) phenotypes and rate of adolescent development. Ann Hum Biol. 1989;16(2):131-146. doi:10.1080/03014468700006982

30. Kim UK, Jorgenson E, Coon H, Leppert M, Risch N, Drayna D. Positional cloning of the human quantitative trait locus underlying taste sensitivity to phenylthiocarbamide. Science. 2003;299 (5610):1221-1225. doi:10.1126/science. 1080190

31. Whissell-Buechy D. Effects of age and sex on taste sensitivity to phenylthiocarbamide (PTC) in the Berkeley guidance sample. Chem Senses. 1990;15(1):39-57. doi:10.1093/chemse/15.1.39

32. Adappa ND, Howland TJ, Palmer JN, et al. Genetics of the taste receptor T2R38 correlates with chronic rhinosinusitis necessitating surgical intervention. Int Forum Allergy Rhinol. 2013;3(3):184-187. doi:10.1002/alr.21140
33. Mfuna Endam L, Filali-Mouhim A, Boisvert P, Boulet LP, Bosse Y, Desrosiers M. Genetic variations in taste receptors are associated with chronic rhinosinusitis: a replication study. Int Forum Allergy Rhinol. 2014;4(3):200-206. doi:10.1002/alr.21275

34. Adappa ND, Zhang Z, Palmer JN, et al. The bitter taste receptor T2R38 is an independent risk factor for chronic rhinosinusitis requiring sinus surgery. Int Forum Allergy Rhinol. 2014;4(1):3-7. doi:10. 1002/alr.21253

35. Adappa ND, Farquhar D, Palmer JN, et al. TAS2R38 genotype predicts surgical outcome in nonpolypoid chronic rhinosinusitis. Int Forum Allergy Rhinol. 2016;6(1):25-33. doi:10.1002/alr.21666

36. Adappa ND, Truesdale CM, Workman AD, et al. Correlation of T2R38 taste phenotype and in vitro biofilm formation from nonpolypoid chronic rhinosinusitis patients. Int Forum Allergy Rhinol. 2016;6(8):783-791. doi:10.1002/alr.21803

37. Rom DI, Christensen JM, Alvarado R, Sacks R, Harvey RJ. The impact of bitter taste receptor genetics on culturable bacteria in chronic rhinosinusitis. Rhinology. 2017;55(1):90-94. doi:10.4193/ Rhin 16.181

38. Zayzafoon M. Calcium/calmodulin signaling controls osteoblast growth and differentiation. J Cell Biochem. 2006;97(1):56-70. doi:10.1002/jcb.20675

39. Theman TA, Collins MT. The role of the calcium-sensing receptor in bone biology and pathophysiology. Curr Pharm Biotechnol. 2009;10 (3):289-301. doi:10.2174/138920109787847538

40. Goltzman D, Hendy GN. The calcium-sensing receptor in bonemechanistic and therapeutic insights. Nat Rev Endocrinol. 2015;11 (5):298-307. doi:10.1038/nrendo.2015.30

41. Fromigue O, Hay E, Barbara A, et al. Calcium sensing receptor-dependent and receptor-independent activation of osteoblast replication and survival by strontium ranelate. J Cell Mol Med. 2009;13(8B):2189-2199. doi:10.1111/j.1582-4934.2008.00673.x

42. Pi M, Garner SC, Flannery P, Spurney RF, Quarles LD. Sensing of extracellular cations in CasR-deficient osteoblasts. Evidence for a novel cation-sensing mechanism. J Biol Chem. 2000;275 (5):3256-3263. doi:10.1074/jbc.275.5.3256

43. Marie PJ. The calcium-sensing receptor in bone cells: a potential therapeutic target in osteoporosis. Bone. 2010;46(3):571-576. doi:10.1016/j.bone.2009.07.082

44. Tordoff MG. Calcium: taste, intake, and appetite. Physiol Rev. 2001;81(4):1567-1597. doi:10.1152/physrev.2001.81.4.1567

45. Hwang SY, Putney JW Jr. Calcium signaling in osteoclasts. Biochim Biophys Acta. 2011;1813(5):979-983. doi:10.1016/j.bbamcr.2010.11. 002

46. Yamaguchi M, Hamamoto R, Uchiyama S, Ishiyama K. Effects of flavonoid on calcium content in femoral tissue culture and parathyroid hormone-stimulated osteoclastogenesis in bone marrow culture in vitro. Mol Cell Biochem. 2007;303(1-2):83-88. doi:10.1007/s11010007-9458-x

47. Meyerhof W, Batram C, Kuhn C, et al. The molecular receptive ranges of human TAS2R bitter taste receptors. Chem Senses. 2010;35(2):157-170. doi:10.1093/chemse/bjp092

48. Lacativa PG, Farias ML. Osteoporosis and inflammation. Arq Bras Endocrinol Metabol. 2010;54(2):123-132. doi:10.1590/S0004-2730 2010000200007

49. Abu-Amer Y, Darwech I, Clohisy JC. Aseptic loosening of total joint replacements: mechanisms underlying osteolysis and potential therapies. Arthritis Res Ther. 2007;9(Suppl 1):S6. doi:10.1186/ar2170

50. Ruegg C. Leukocytes, inflammation, and angiogenesis in cancer: fatal attractions. J Leukoc Biol. 2006;80(4):682-684. doi:10.1189/ jlb.0606394

51. Roca H, McCauley LK. Inflammation and skeletal metastasis. Bonekey Rep. 2015;4:706. doi:10.1038/bonekey.2015.75

52. El-Jawhari JJ, Jones E, Giannoudis PV. The roles of immune cells in bone healing; what we know, do not know and future perspectives. Injury. 2016;47(11):2399-2406. doi:10.1016/j.injury.2016.10.008 
53. Van't Hof RJ, Ralston SH. Nitric oxide and bone. Immunology 2001;103(3):255-261. doi:10.1046/j.1365-2567.2001.01261.x

54. Pilbeam C. Prostaglandins and bone. In: Handb Exp Pharmacol. Berlin, Heidelberg: Springer; 2019.

55. Tian XY, Zhang Q, Zhao R, et al. Continuous PGE2 leads to net bone loss while intermittent PGE2 leads to net bone gain in lumbar vertebral bodies of adult female rats. Bone. 2008;42(5):914-920. doi:10.1016/j.bone.2007.12.228

56. Ragipoglu D, Dudeck A, Haffner-Luntzer M, et al. The role of mast cells in bone metabolism and bone disorders. Front Immunol. 2020;11:163. doi:10.3389/fimmu.2020.00163

57. Brady RA, Leid JG, Calhoun JH, Costerton JW, Shirtliff ME. Osteomyelitis and the role of biofilms in chronic infection. FEMS Immunol Med Microbiol. 2008;52(1):13-22. doi:10.1111/j.1574695X.2007.00357.x

58. Gomez-Barrena E, Esteban J, Medel F, et al. Bacterial adherence to separated modular components in joint prosthesis: a clinical study. J Orthop Res. 2012;30(10):1634-1639. doi:10.1002/jor.22114

59. Esteban J, Cordero-Ampuero J. Treatment of prosthetic osteoarticular infections. Expert Opin Pharmacother. 2011;12(6):899-912. doi:10. 1517/14656566.2011.543676

60. Lee RJ, Kofonow JM, Rosen PL, et al. Bitter and sweet taste receptors regulate human upper respiratory innate immunity. $J$ Clin Invest. 2014;124(3):1393-1405. doi:10.1172/JCI72094

61. Varoga D, Tohidnezhad M, Paulsen F, et al. The role of human beta-defensin-2 in bone. J Anat. 2008;213(6):749-757. doi:10.1111/ j.1469-7580.2008.00992.x

62. Varoga D, Wruck CJ, Tohidnezhad M, et al. Osteoblasts participate in the innate immunity of the bone by producing human beta defensin-3. Histochem Cell Biol. 2009;131(2):207-218. doi:10.1007/s00418-0080522-8

63. Zhu C, Qin H, Cheng T, et al. Staphylococcus aureus supernatant induces the release of mouse beta-defensin-14 from osteoblasts via the p38 MAPK and NF-kappaB pathways. Int J Mol Med. 2013;31 (6):1484-1494. doi:10.3892/ijmm.2013.1346
64. Thilakarathna SH, Rupasinghe HP. Flavonoid bioavailability and attempts for bioavailability enhancement. Nutrients. 2013;5(9):33 67-3387. doi:10.3390/nu5093367

65. Roca-Ferrer J, Pujols L, Perez-Gonzalez M, et al. Superior effect of MP-AzeFlu than azelastine or fluticasone propionate alone on reducing inflammatory markers. Allergy Asthma Clin Immunol. 2018;14 (1):86. doi:10.1186/s13223-018-0311-4

66. Ekstedt S, Kumlien Georen S, Cardell LO. Effects of MP-AzeFlu enhanced by activation of bitter taste receptor TAS2R. Allergy Asthma Clin Immunol. 2020;16(1):45. doi:10.1186/s13223-020-00438-w

67. Deloose E, Janssen P, Corsetti M, et al. Intragastric infusion of denatonium benzoate attenuates interdigestive gastric motility and hunger scores in healthy female volunteers. Am $J$ Clin Nutr. 2017;105(3):580-588. doi:10.3945/ajen.116.138297

68. Deloose E, Corsetti M, Van Oudenhove L, Depoortere I, Tack J. Intragastric infusion of the bitter tastant quinine suppresses hormone release and antral motility during the fasting state in healthy female volunteers. Neurogastroenterol Motil. 2018;30(1):e13171. doi:10.11 $11 / \mathrm{nmo} .13171$

69. Wolfle U, Elsholz FA, Kersten A, Haarhaus B, Muller WE, Schempp CM. Expression and functional activity of the bitter taste receptors TAS2R1 and TAS2R38 in human keratinocytes. Skin Pharmacol Physiol. 2015;28(3):137-146. doi:10.1159/000367631

70. Schempp C, Woelfle U, Inventors; Bitter taste receptor agonists for topical use. US patent WO2013112865A12013.

71. Ezra A, Golomb G. Administration routes and delivery systems of bisphosphonates for the treatment of bone resorption. Adv Drug Deliv Rev. 2000;42(3):175-195. doi:10.1016/S0169-409X(00)00061-2

72. Ford CA, Cassat JE. Advances in the local and targeted delivery of anti-infective agents for management of osteomyelitis. Expert Rev Anti Infect Ther. 2017;15(9):851-860. doi:10.1080/14787210.2017.1372192

73. Carbone EJ, Rajpura K, Allen BN, Cheng E, Ulery BD, Lo KW. Osteotropic nanoscale drug delivery systems based on small molecule bone-targeting moieties. Nanomedicine. 2017;13(1):37-47. doi:10.10 16/j.nano.2016.08.015
Drug Design, Development and Therapy

\section{Publish your work in this journal}

Drug Design, Development and Therapy is an international, peerreviewed open-access journal that spans the spectrum of drug design and development through to clinical applications. Clinical outcomes, patient safety, and programs for the development and effective, safe, and sustained use of medicines are a feature of the journal, which has also been accepted for indexing on PubMed Central. The manuscript management system is completely online and includes a very quick and fair peer-review system, which is all easy to use. Visit http://www. dovepress.com/testimonials.php to read real quotes from published authors. 